\title{
Model study of ground magnetic signatures of traveling convection vortices
}

\author{
L. Zhu, P. Gifford, J. J. Sojka, and R. W. Schunk \\ Center for Atmospheric and Space Science, Utah State University, Logan
}

\begin{abstract}
We conducted a model study of ground magnetic signatures of traveling convection vortices (TCVs) that included both the ionospheric conductivity enhancement associated with the TCVs and the ground induction effect. We found that the localized conductivity enhancement can cause a significant distortion of the TCV current system and lead to a distortion of the ground magnetic disturbance patterns. The patterns of all three magnetic components are asymmetric, mainly in the E-W direction, and the patterns of the $Z$ component show the strongest asymmetry (20-30\%). We also found that the effect of induction currents on ground magnetic signatures of the TCVs is insignificant (less than 5\%). The results show that because of the presence of localized conductivity enhancements the polarity and speed of the TCVs can significantly influence the distortion features of ground magnetic patterns. The upward and downward current filaments of a TCV with a clockwise leading convection cell can wrap with each other, resulting in a rotation of the whole ground magnetic disturbance pattern. This rotation feature is most significant when the speed of the TCVs is high.
\end{abstract}

\section{Introduction}

Traveling convection vortices (TCVs) are the localized convection cells that appear in the prenoon or postnoon sectors of the high-latitude ionosphere and move in a predominantly antisunward direction [Friis-Christensen et al., 1988; Glassmeier et al., 1989; Heikkila et al., 1989; McHenry et al., 1990a, b; Kivelson and Southwood, 1991; Glassmeier, 1992; Glasmeier and Heppner, 1992; Southwood and Kivelson, 1993; Vo et al., 1994; Lühr and Blawert, 1994; Bristow et al., 1995]. They have also been called magnetic impulse events [Lanzerotti et al., 1986, 1991] when observed with a single magnetometer. Most of the observed TCVs consist of a pair of convection vortices with opposite rotating directions that are aligned predominantly in the E-W direction, but recent observations indicate that vortices with three cells are not uncommon [Sibeck, 1995; Zesta et al., 1995]. The E-W extent of the TCVs ranges from several hundreds of kilometers to $1000 \mathrm{~km}$, and the $\mathrm{N}-\mathrm{S}$ extent is about $500 \mathrm{~km}$. Most of the TCVs move toward the nightside with speeds in a range of 3-6 $\mathrm{km} / \mathrm{s}$, but some cases with extremely low (several hundreds of meters per second) and high (more than $20 \mathrm{~km} / \mathrm{s}$ ) speeds were also reported. Some TCVs do not have a constant speed and/or constant intensity in time, and both can vary on a very short timescale. Although single TCV structures are more common, a continuous series of TCVs was also observed to move in the antisunward direction for a period of several hours, with about a 15-min time interval between TCVs. Field-aligned current filaments are associated with the convection vortices, and the magnitude of the currents is estimated to be of the order of $1 \mu \mathrm{A} / \mathrm{m}^{2}$. Corresponding to the convection vortices with alternating rotation directions, the neighboring field-aligned currents in the TCVs flow in opposite directions. Field-aligned

Copyright 1997 by the American Geophysical Union.

Paper number 96JA03682.

0148-0227/97/96JA-03682\$09.00 current associated with the leading cell (tailward cell) of the twin or triple vortices can be either upward or downward. The characteristic energy of the precipitation in the upward current cell is about $1 \mathrm{keV}$, and short-lived auroral brightenings can be associated with the TCVs. The TCVs can cause significant ionospheric effects, including ion and electron temperature enhancements, ion temperature anisotropies, $\mathrm{O}^{+}$to $\mathrm{NO}^{+}$ composition changes, and elevated plasma scale heights [Schunk et al., 1994].

At present, the prevailing view is that the traveling convection vortices are not a result of flux transfer events (FTEs) at the dayside magnetopause, but their exact cause is still controversial. Friis-Christensen et al. [1988] suggested that the TCVs are due to sudden changes in the solar wind pressure or the interplanetary magnetic field (IMF). Heikkila et al. [1989] suggested that the TCVs are due to penetration of solar wind plasma clouds, while McHenry et al. [1990b] proposed that the vortices are due to a Kelvin-Helmholtz instability at the low-latitude boundary layers (LLBL). Recently, it has also been suggested that the TCVs are caused by solar wind dynamic pressure pulses [Sibeck, 1990; Kivelson and Southwood, 1991; Glasmeier and Heppner, 1992], and numerical simulations of the TCVs based on the theory of solar wind dynamic pressure pulses have been conducted [Lysak and Lee, 1992].

The current systems of the TCVs can cause significant ground magnetic disturbances, and the study of ground magnetic signatures of the TCVs has been the most important component of the TCV studies. The observed magnetic disturbance patterns normally appear to be distorted and asymmetric, and Friis-Christensen et al. [1988] have speculated that the distortion might be related to the conductivity gradient caused by the TCVs' precipitation and the ground induction.

The results of theoretical modeling of ground magnetic disturbances associated with localized ionospheric current systems have been reported in several previous studies 「McHenry and Clauer, 1987; Zhu and Kan, 1989; Wei and Lee, 
1990; Chaston et al., 1993]. However, all these modeling studies mainly concerned the localized current systems associated with FTEs, and none of them included the effects of the conductivity enhancement and ground induction.

When the magnetosphere-originated convection vortices reach the ionosphere, a significant conductivity enhancement caused by the precipitation associated with the upward fieldaligned currents of the TCVs can change the current closure in the ionosphere and distort the TCVs' ionospheric current system. Hence a distorted magnetic disturbance pattern would be expected on the ground. The distortion should be most severe for the case in which the upward current cell is the leading cell, because the high speed of the TCVs allows a significant portion of the downward current cell to intrude into the enhanced conductivity region left by the upward current cell before the conductivity enhancement subsides due to ionospheric recombination. Then the downward field-aligned current can also be distorted by the conductivity gradient, and the enhanced conductivity distribution, in turn, can be disturbed by the downward current cell through plasma transport. Both effects lead to a further distortion of the whole TCV ionospheric current system and the ground magnetic disturbance pattern. At the same time a distorted ground induction current system, due to a distorted ionospheric current system, may make a further contribution to the distortion of the ground magnetic pattern associated with the TCV.

On the basis of the above considerations we conducted a series of numerical studies of the ground magnetic signatures of the TCVs, in which the ionospheric conductivity enhancement associated with the TCVs and ground induction effect were included. Our goal was to determine how the localized conductivity gradient and ground induction contribute to the distortion of the ground magnetic disturbance pattern of a TCV and how the various ionospheric characteristics of the TCV (such as speed and polarity) affect the features of the ground magnetic pattern.

\section{Formulation}

The ionosphere is assumed to be a slab with heightintegrated conductivities, and the Earth magnetic field lines are assumed to be perpendicular to the ionosphere. In the modeling we focus on the TCVs occurring in the prenoon sector. Therefore the numerical domain is located in the morning sector of the high-latitude ionosphere. The numerical domain is $3600 \mathrm{~km}$ long in the $\mathrm{E}-\mathrm{W}(X)$ direction and $1100 \mathrm{~km}$ wide in the N-S $(Y)$ direction. Actually, with some sign changes the model can also be used to study the magnetic signatures of the TCVs occurring in the postnoon sector. A Cartesian coordinate system is adopted in the model in which the $X$ axis points to the west, the $Y$ axis points to the south, and the $Z$ axis is upward.

In the model the magnetosphere-originated convection vortices are externally imposed, which implies an assumption of constant voltage source for the TCVs. Since the TCVs are due to the direct interactions between the solar wind and magnetosphere near the magnetopause, the solar wind inertia is sufficiently large that the loading effect of the ionosphere on the TCVs' source regions should be insignificant on the zeroth order. The rational of the assumption could be severely violated in the inner magnetosphere where the plasma inertia is small (e.g., the plasma sheet). In such a situation the loading of the ionosphere can make a noticeable modification to the magnetospheric convection. To precisely simulate the electrodynamics of the TCVs, one has to treat them in a timedependent magnetosphere-ionosphere coupling system that further includes the self-consistent ionospheric current closure in the magnetospheric source regions and a dynamic magnetosphere, which is far beyond the goals of this paper.

When the electric fields associated with the traveling convection vortices $(\mathbf{E})$, which can be either two or three cells, reach the ionosphere, the corresponding ionospheric horizontal current, I, can be expressed as

$$
\mathbf{I}=\Sigma_{P} \mathbf{E}+\Sigma_{H} \hat{\mathbf{B}}_{o} \times \mathbf{E}
$$

where $\mathbf{B}_{o}$ is the unit vector of the magnetic field and $\Sigma_{p}$ and $\Sigma_{H}$ are the height-integrated Pedersen and Hall conductivities, which include both the background ionospheric conductance and the enhanced conductance caused by the TCVs' precipitation. From the current continuity equation one can then obtain the field-aligned current

$$
J_{\|}(x, y)=-\nabla \cdot \mathbf{I}
$$

The precipitation associated with the upward field-aligned currents of TCVs can cause a significant enhancement of the ionospheric conductance in a localized region [Sibeck, 1995], and then the conductances in equation (1) are not uniform. We use the continuity equation to calculate the conductance enhancement associated with the TCVs, i.e.,

$$
\frac{\partial N}{\partial t}+\nabla \cdot(N \mathrm{~V})=\frac{Q J_{\|}}{e}+\frac{J_{\|}}{e H}+S_{o}-\beta N^{2}
$$

where $N$ is the electron number density in the ionosphere, $\mathbf{V}$ is the convection velocity, $Q$ is the ionization coefficient, which will be defined later, $H$ is the effective height of the conducting ionosphere, $\beta$ is the recombination coefficient, and $S_{o}$ is the background ionization source term, which can be approximately determined by the background electron density $N_{o}$, i.e.,

$$
S_{o}=\beta N_{o}^{2}
$$

Note that in equation (3) the upward field-aligned current has a positive value.

The first term on the right-hand side of equation (3) is the ionization term related to the precipitation associated with the field-aligned currents, and the second term is the electron number flux carried by the field-aligned currents. In the model the ionization coefficient $Q$ is defined as

$$
\begin{aligned}
& Q=0 \quad J_{\|} \quad \text { downward } \\
& Q=\gamma^{\prime}\left|J_{\|}\right| J_{\|} \quad \text { upward }
\end{aligned}
$$

where $\gamma^{\prime}$, which is 0.8 (ions $\left.\mathrm{m}\right) /($ electron $\mathrm{A}$ ) in all the simulations, is a parameter that depends on the $J_{\|}-\phi_{\|}$ relationship [Fridman and Lemaire, 1980]. As indicated by equation (5), the ionization caused by the precipitation associated with the downward field-aligned current has been assumed to be insignificant, and only the ionization effects of the precipitating electrons associated with the upward fieldaligned current are considered. 
By assuming a constant ratio $R=\Sigma_{H} / \Sigma_{P}$, which is 1 in the model, and using an approximate expression for the heightintegrated Hall conductivity

$$
\Sigma_{H}=\frac{N e H}{B_{o}},
$$

equation (3) can be rewritten as

$$
\begin{aligned}
& \frac{\partial \Sigma_{H}}{\partial t}=\frac{-(Q H+1)}{B_{o}} \nabla \cdot\left(\Sigma_{P} \mathbf{E}_{i}+\Sigma_{H} \mathbf{b}_{o} \times \mathbf{E}_{i}\right) \\
& +\frac{e H}{B_{o}} S_{o}-\frac{\beta B_{o}}{e H} \Sigma_{H}^{2}+\nabla \Sigma_{H} \cdot \frac{\mathbf{b}_{o} \times \mathbf{E}_{i}}{B_{o}}
\end{aligned}
$$

This is the working equation in the model to calculate the conductivity enhancement caused by the TCVs.

To get the ground magnetic signatures of the TCVs, we use the Biot-Savart law,

$$
d \mathbf{B}=\frac{\mu_{o}}{4 \pi r^{3}}(\mathbf{J} \times \mathbf{r}) d V
$$

In all previous theoretical modeling of ground magnetic disturbances associated with the FTEs' ionospheric current system [McHenry and Clauer, 1987; Zhu and Kan, 1989; Chaston et al., 1993], a uniform ionospheric conductance was assumed, and only the magnetic effect of the Hall current was considered. This was based on Fukushima's [1969] theorem that if the conductance is uniform and the field-aligned current is perpendicular to the ionosphere, the ground magnetic perturbation is produced exclusively by the Hall current. The magnetic perturbations produced by the Pedersen current and the field-aligned current cancel each other. In our model the assumption of a uniform ionospheric conductance is removed, and the localized conductance enhancement associated with the TCVs' is included, which is one of the major differences between our model and the previous work. Therefore, when we calculate the ground magnetic perturbation, all three components of the TCVs current system are included, and equation (8) can be rewritten as

$$
d \mathbf{B}\left(\mathbf{r}_{o}\right)=\frac{\mu_{o}}{4 \pi r^{\prime 2}}\left\{\left[\mathbf{I}_{H}(\mathbf{r})+\mathbf{I}_{P}(\mathbf{r})\right] \times \hat{\mathbf{r}}^{\prime} d x d y+\mathbf{J}_{\|}(\mathbf{r}) \times \hat{\mathbf{r}}^{\prime} d x d y d z\right\}
$$

where $\mathbf{r}_{o}$ is the observation point on the ground, $\mathbf{r}$ is the source point in the ionosphere, $r^{\prime}$ is the magnitude of $\mathbf{r}^{\prime}$ while $\mathbf{r}^{\prime}=\mathbf{r}_{o}-\mathbf{r}, \hat{\mathbf{r}}^{\prime}$ is the unit vector of $\mathbf{r}^{\prime}$, and $I_{H}$ and $I_{P}$ are the ionospheric Hall and Pedersen currents, respectively. Then the integrated ground magnetic perturbation can be expressed as

$$
\begin{aligned}
& \mathbf{B}\left(x_{o}, y_{o}\right)=\iint \frac{\mu_{o}}{4 \pi r^{\prime 2}}\left(\mathbf{I}_{H}+\mathbf{I}_{P}\right) \times \hat{\mathbf{r}}^{\prime} d x d y+ \\
& \iiint \frac{\mu_{o}}{4 \pi r^{\prime 2}} \mathbf{J}_{\|} \times \hat{\mathbf{r}}^{\prime} d x d y d z
\end{aligned}
$$

where $x_{o}$ and $y_{o}$ are the $x$ and $y$ components of $\mathbf{r}_{o}$, respectively. The horizontal area of integration is the entire ionospheric domain specified previously, and the integration in the $Z$ direction goes up to the altitude of $2000 \mathrm{~km}$.

To estimate the effect of the induction current on the ground magnetic signature of a TCV, we have to make some assumptions. When a uniform Earth conductivity is assumed, the Earth can be simplified as an infinitely conducting sphere covered by a perfect insulator layer that is at least $250 \mathrm{~km}$ thick [Chapman, 1919; Kisabeth and Rostoker, 1977; Campbell, 1987]. In our model the magnetic effect of the Earth induction current is calculated from image currents of the TCVs' ionospheric current system, which flows in the Earth conducting sphere. In reality, the structure of the Earth is quite inhomogeneous. But the estimation of the effect of the Earth's inhomogeneity on the induction currents of the TCV is difficult and is beyond the scope of this study.

\section{Results}

In our modeling, typical localized magnetospheric convection cells are imposed on the morning sector of the high-latitude ionosphere, and the convection cells then move toward the nightside with a constant speed. The average energy of the precipitating electrons associated with upward fieldaligned current is assumed to be about $1 \mathrm{keV}$. The distance between the ionospheric slab and ground is assumed to be 140 $\mathrm{km}$, and the Earth's curvature is ignored.

\subsection{Effects of Conductivity Enhancement}

To study how the ionospheric conductivity enhancement caused by TCV precipitation affects the ground magnetic disturbance patterns of the TCVs, we need a standard case for which the conductivity enhancement and induction currents are not included. Such a case is shown in Figure 1, where the background ionospheric conductance is uniform ( 3 mhos). The top panel shows a pair of imposed magnetospheric convection cells moving toward the nightside (westward) with a constant speed of $4 \mathrm{~km} / \mathrm{s}$. The potential difference between the two convection cells is $15 \mathrm{kV}$. Since the background conductivity is uniform and no conductivity enhancement associated with TCV precipitation is included, the current closure of the TCV in the ionosphere is symmetric. A pair of symmetric field-aligned currents associated with the TCV are shown in the left middle panel of Figure 1, where solid lines mean upward field-aligned currents.

The rest of the panels in Figure 1 show the ground magnetic disturbance patterns of the $H, D$, and $Z$ components associated with the TCV. Note that even though the model includes the contributions from all three components of the TCV current system (Hall, Pedersen, and field-aligned currents) when it calculates the ground magnetic disturbances, the resulting magnetic patterns are the same as those associated with the Hall current only in this particular case. This finding is consistent with Fukushima's [1969] theorem. This equivalence will not hold when the ionospheric conductivity enhancement associated with the TCV is included. The results shown in Figure 1 indicate that when the conductivity is uniform the ground magnetic disturbance patterns are all symmetric for a pair of externally imposed symmetric convection cells.

Figure 2 show the case in which the effect of the localized conductivity enhancement is included. In this case the background ionospheric conductivity (uniform, 3 mhos), polarity (downward field-aligned current leads), and the speed of the TCV (4 km/s westward) are the same as those for the case shown in Figure 1. The top panel in Figure 2 shows the localized conductivity enhancement due to the TCV precipitation. Since we only consider the ionization due to the energetic precipitating electrons associated with upward 


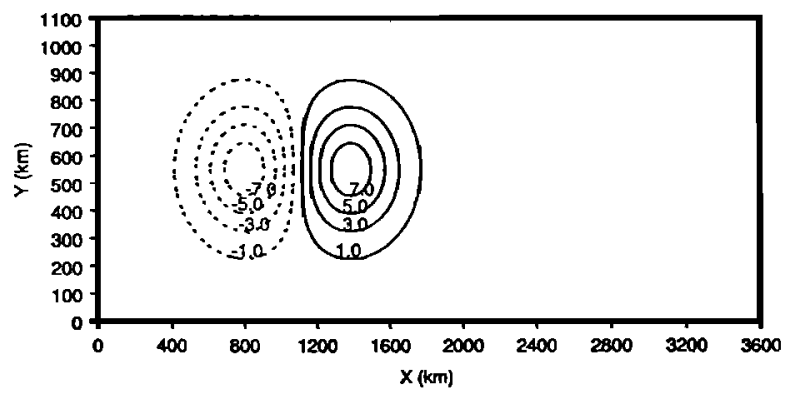

FIELO-ALIGNED CURRENT (UAM $\left.{ }^{2}\right)$

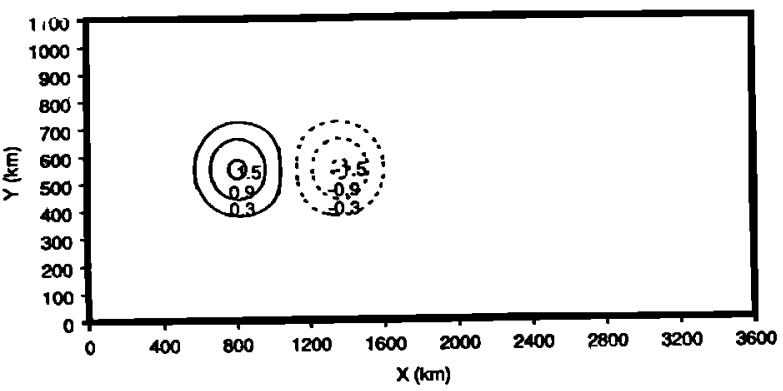

D COMPONENT (nT)

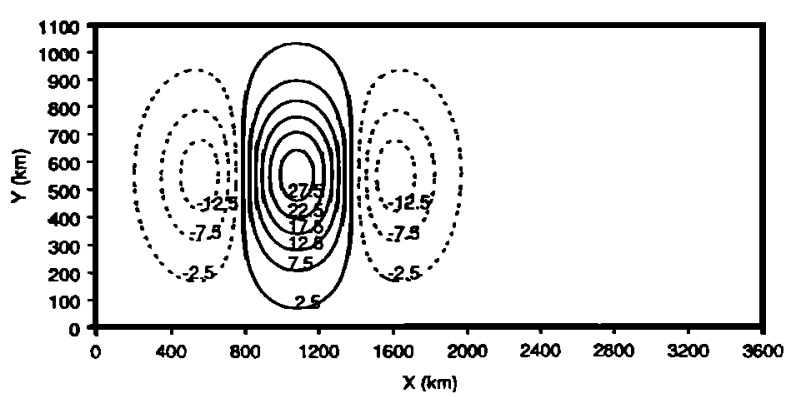

H COMPONENT (nT)

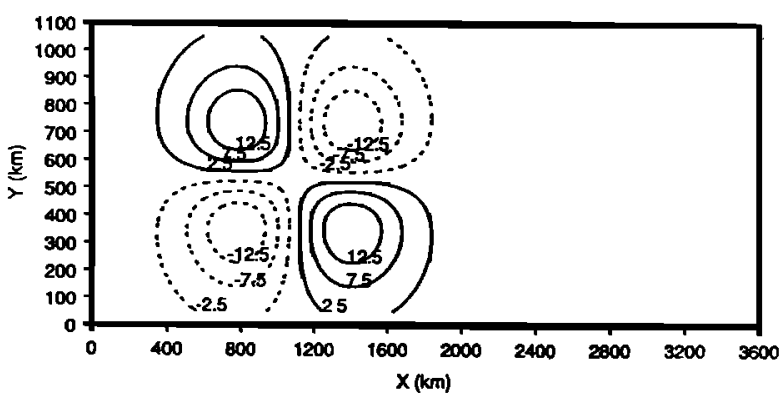

COMPONENT (nT)

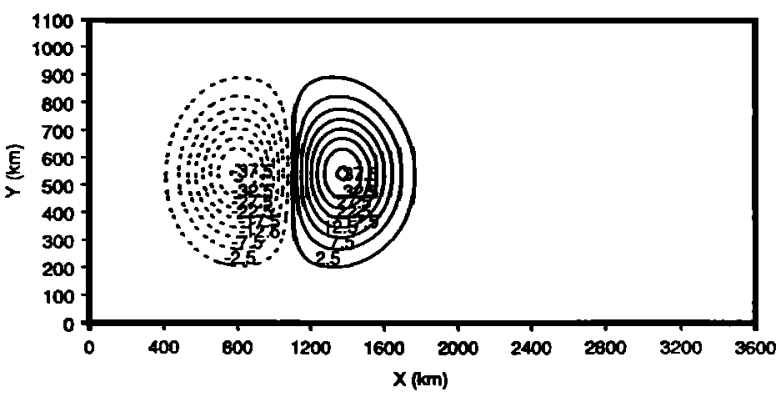

Figure 1. TCV signatures for the case in which the effects of conductivity enhancement and ground induction are excluded. The convection speed of the TCV is $4 \mathrm{~km} / \mathrm{s}$, and the background ionospheric conductance is uniform ( 3 mhos). Counterclockwise convection cell (downward current) leads.

current, the resulting single localized high-conductivity region is expected. Because of the conductivity gradient the current closure of the TCV in the ionosphere is no longer symmetric, and both the horizontal and field-aligned currents can be distorted. In this particular case the distortion mainly occurs with the upward current cell, since the downward current cell is in the leading position, and the speed of the TCV is high. The distorted field-aligned current distribution is shown in the left middle panel in Figure 2. As can be seen, the upward fieldaligned current is severely distorted because of the conductivity gradient.

The rest of Figure 2 shows the ground magnetic disturbance patterns of the $H, D$, and $Z$ components. Figure 2 clearly shows that the patterns of all three components are now asymmetric in the E-W direction, with stronger magnetic disturbances on the side of the upward field-aligned current. The $Z$ component pattern shows the strongest asymmetry, about $20 \%$. Because the distorted TCV current system also is asymmetric in the N-S direction, a slight N-S asymmetry can be seen in the ground magnetic patterns.

As we mentioned before, the average energy of the precipitating electrons associated with the upward field-aligned current was assumed to be $1 \mathrm{keV}$. When the average energy of the precipitating electrons is increased, the localized conductivity enhancement can be greater, and then a stronger asymmetry can be expected in the ground magnetic patterns. We ran several extreme cases with an average energy of about 3 $\mathrm{keV}$ (not shown), and the results displayed $45-50 \%$ E-W asymmetry in the $Z$ component patterns. This finding leads to an important theoretical prediction that a severely distorted magnetic pattern observed on the ground may indicate a TCV with hard precipitation. 

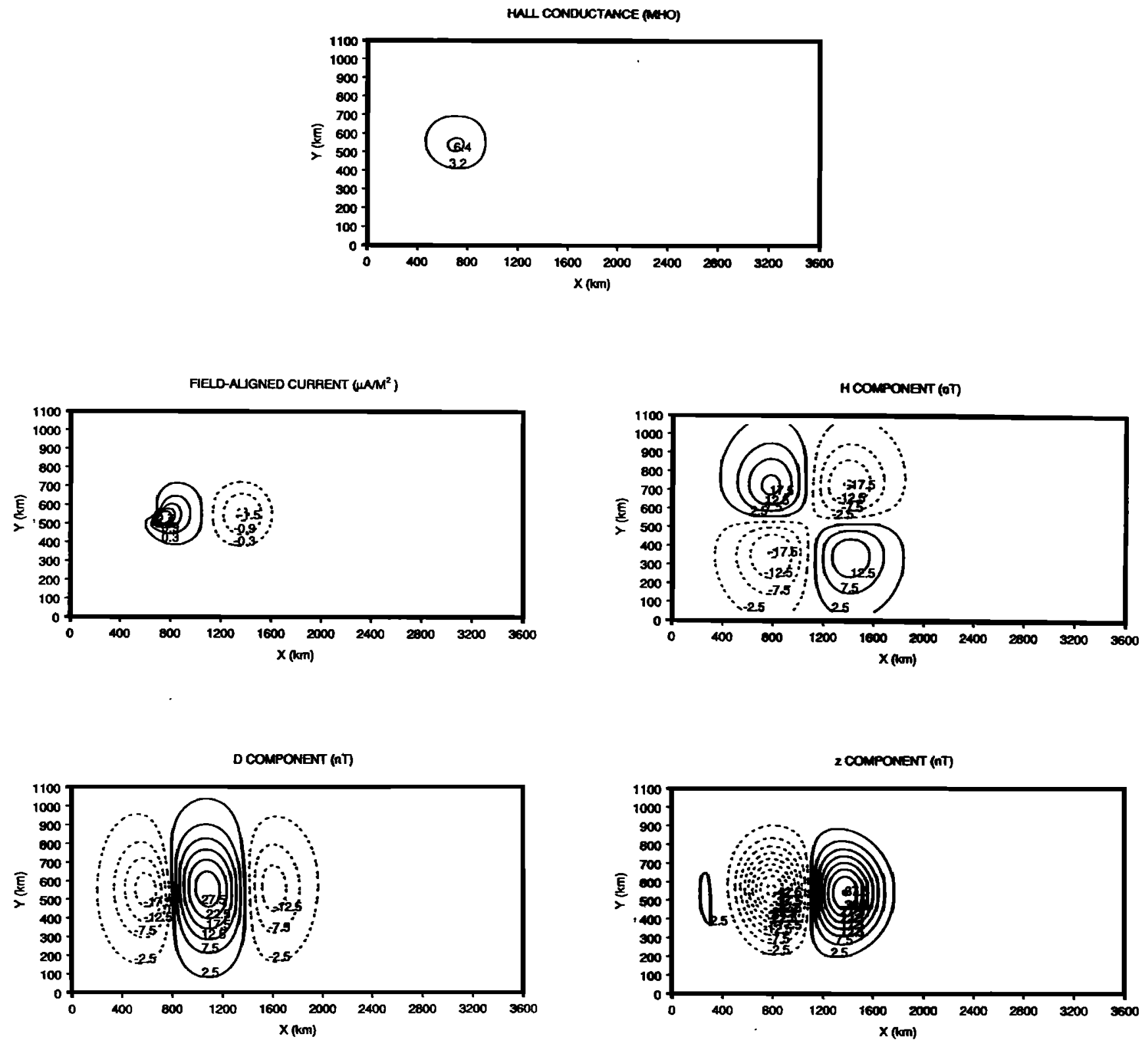

Figure 2. TCV signatures for the case in which the effect of conductivity enhancement is included. The other parameters are the same as for Figure 1.

\subsection{Effects of Induction Currents}

As we mentioned before, the insulator layer of the Earth is $250 \mathrm{~km}$ thick, and the ionospheric slab is $140 \mathrm{~km}$ above the Earth's surface in our modeling. Thus the image currents of the ionospheric horizontal currents are $640 \mathrm{~km}$ below the Earth's surface, and the image currents of the field-aligned currents in the ionosphere extend from that depth toward the center of the Earth.

Figure 3 shows the ground magnetic disturbance patterns of the induction currents for the standard case shown in Figure 1, which are symmetric as expected. Comparing Figures 3 and 1 , we can see that the magnetic disturbance patterns of the induction currents are much more spread than those of the currents in the ionosphere due to a larger distance from the Earth's surface, but the addition of the induction currents does not lead to asymmetric ground magnetic disturbance patterns as long as the ionospheric current system is symmetric. It is also apparent from the comparison that the induction currents actually weaken the disturbances in the $H$ and $D$ components and strengthen the disturbances in the $Z$ component. But the effect of the induction currents is not significant ( $H$ and $D$ components, $4 \% ; Z$ component, $5 \%$ ).

We considered another case (not shown here) in which both the effects of the conductivity enhancement and induction currents are included. It was found that with a distorted TCV current system due to a conductivity enhancement, the induction currents actually slightly reduce the asymmetry in the patterns of the $H$ and $D$ components and increase the asymmetry in the pattern of the $Z$ component, but again the effect is insignificant. 


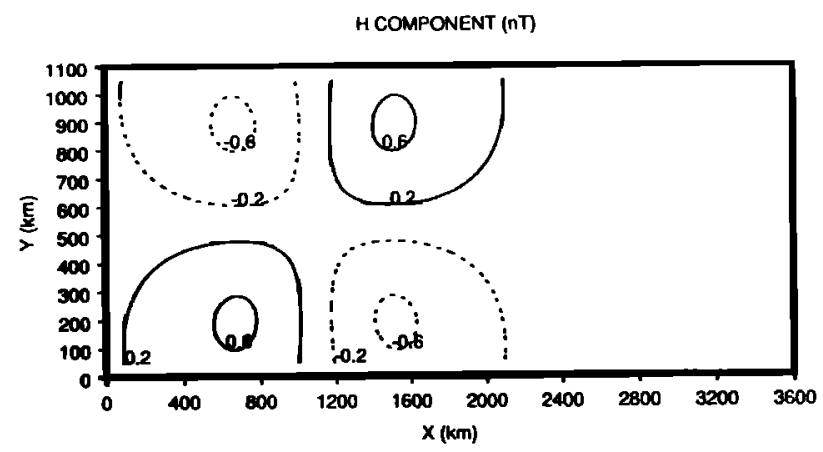

D COMPONENT (nT)

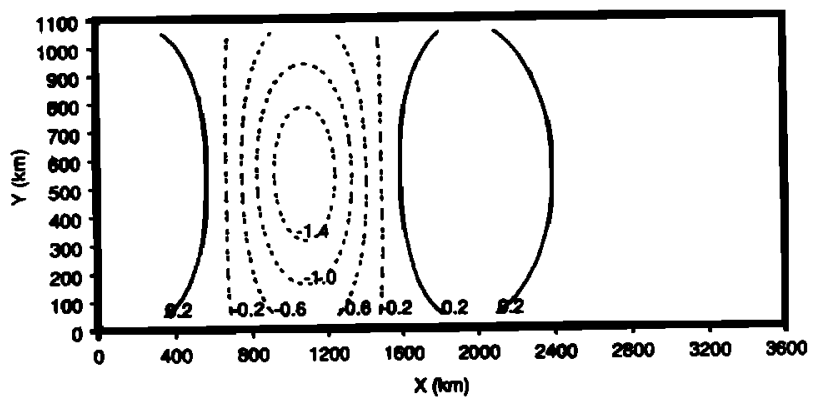

COMPONENT (nT)

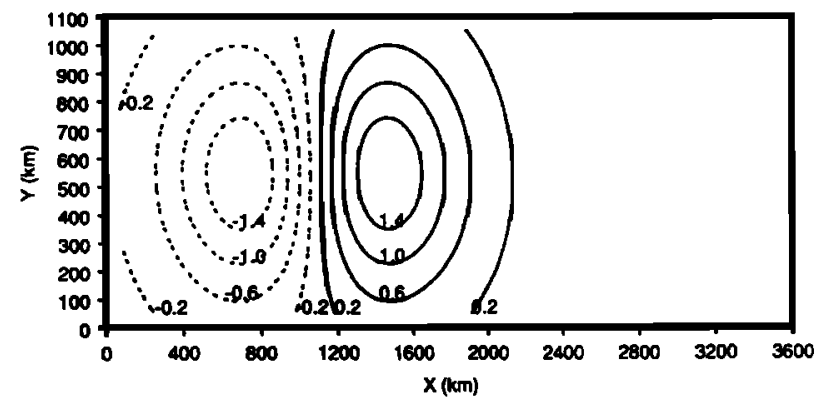

Figure 3. Ground magnetic disturbance patterns of the induction currents for the case shown in Figure 1.

\subsection{The Case Including Conductivity \\ Enhancement, Induction Current, and Background Conductivity Gradient}

To obtain a more realistic ground magnetic disturbance pattern of a TCV, we considered a case in which the localized conductivity enhancement, the induction currents, and the background conductivity gradient due to solar radiation were included. The background conductivity decreased linearly from 3 mhos on the dayside to 1 mho on the nightside, a pattern that approximates the ionospheric conductance distribution in the high-latitude prenoon sector for winter and solar maximum conditions. The polarity (downward field-aligned current leads) and the speed of the TCV (4 km/s westward) are the same as those for the standard case shown in Figure 1.

The results of the run are shown in Figure 4. The top panel shows the conductance distribution, which includes both the enhanced conductance due to the TCV precipitation and the background conductance. The left-middle panel shows the distorted field-aligned current distribution. The rest of the panels show a set of more realistic ground magnetic disturbance patterns of the TCV that could be observed by ground stations for the specific conditions of winter and solar maximum. We can see that the patterns are highly asymmetric in the E-W direction. The asymmetries are about $35 \%$ for the $Z$ component and about $20 \%$ for the $H$ and $D$ components.

\subsection{Effects of TCVs' Polarity}

The variation of the TCV characteristics (e.g., polarity and speed) can certainly lead to various patterns of ground magnetic disturbances. But the inclusion of the conductivity enhancement and induction currents makes the situation more complicated. Taking the polarity of the TCV as an example, if there is no locally enhanced conductivity, a change of the polarity of the TCV only leads to a set of mirrored ground magnetic disturbance patterns. But with the inclusion of the conductivity enhancement the patterns are not mirrored anymore. When the upward current cell is the leading cell, the high speed of the TCV allows a significant portion of the downward current cell to intrude into the enhanced conductivity region left by the upward current cell before the conductivity enhancement subsides due to ionospheric recombination. Then the downward field-aligned current can also be distorted by the conductivity gradient. This situation is different from the case in which the downward current cell is the leading cell, for which only the upward current cell is distorted.

Figure 5 quantitatively shows how the polarity of the TCV influences the ground magnetic patterns. The only difference between the case shown in Figure 5 and the case in the proceeding subsection (Figure 4 ) is the polarity of the TCV. In this case the upward current cell leads instead of the downward current cell in the previous case. As expected, the current system of the counterclockwise convection cell (downward current cell) is now also distorted, since it moves into the enhanced conductivity region. Actually, the two field-aligned current filaments start to wrap around each other. The wrapping leads to a new feature in ground magnetic disturbance patterns, i.e, the rotation of the patterns. This is most evident in the $Z$ component pattern. The rotation of the ground magnetic disturbance patterns for the case in which the counterclockwise convection cell is the leading cell may make a further contribution to the asymmetries of the magnetic disturbances observed by ground-based magnetometers.

\subsection{Effects of TCVs' Speed}

Another ionospheric characteristic of a TCV that can influence the features of their ground magnetic signatures is its speed. Again, this effect become significant only when the 

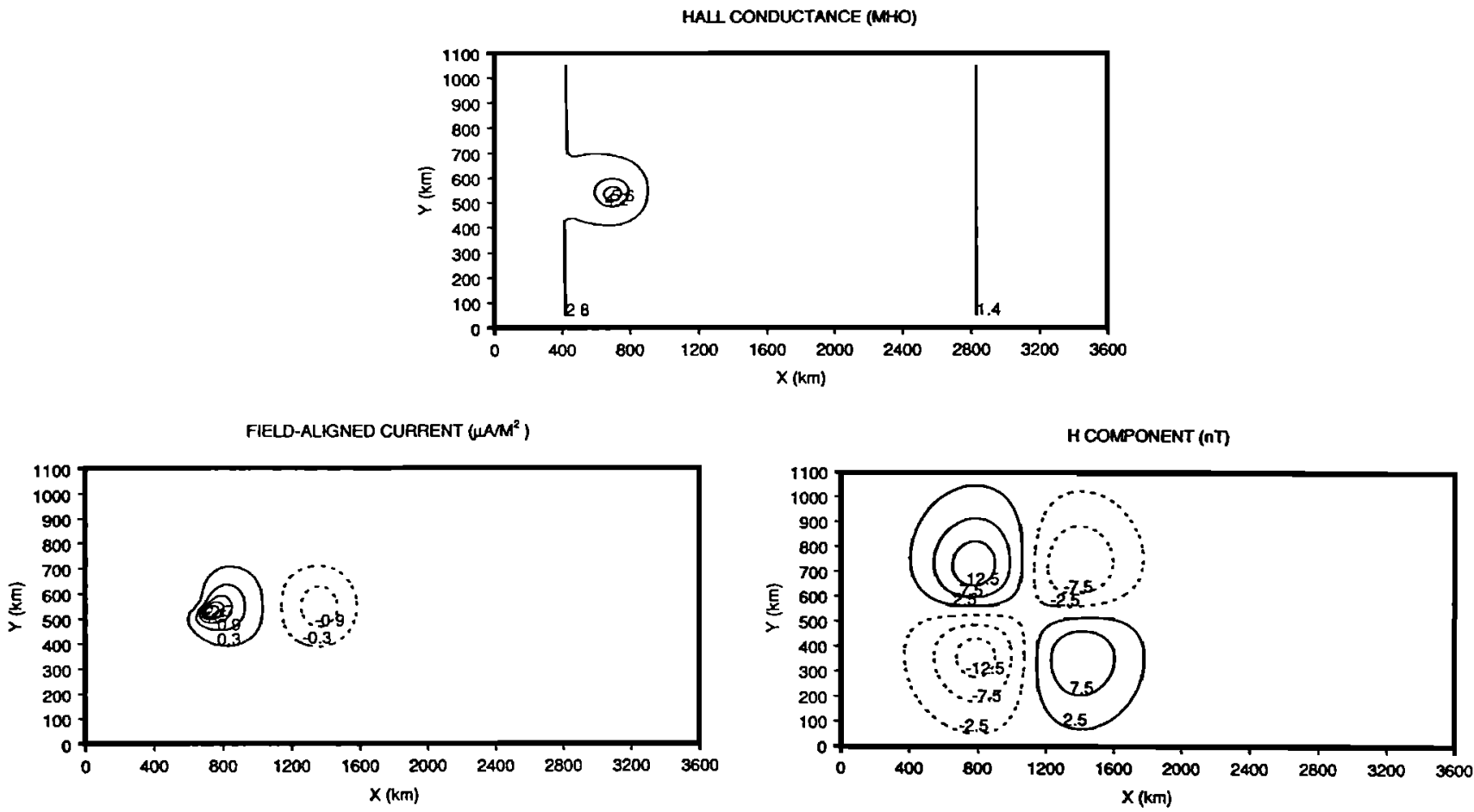

D COMPONENT ( $\mathrm{NT}$
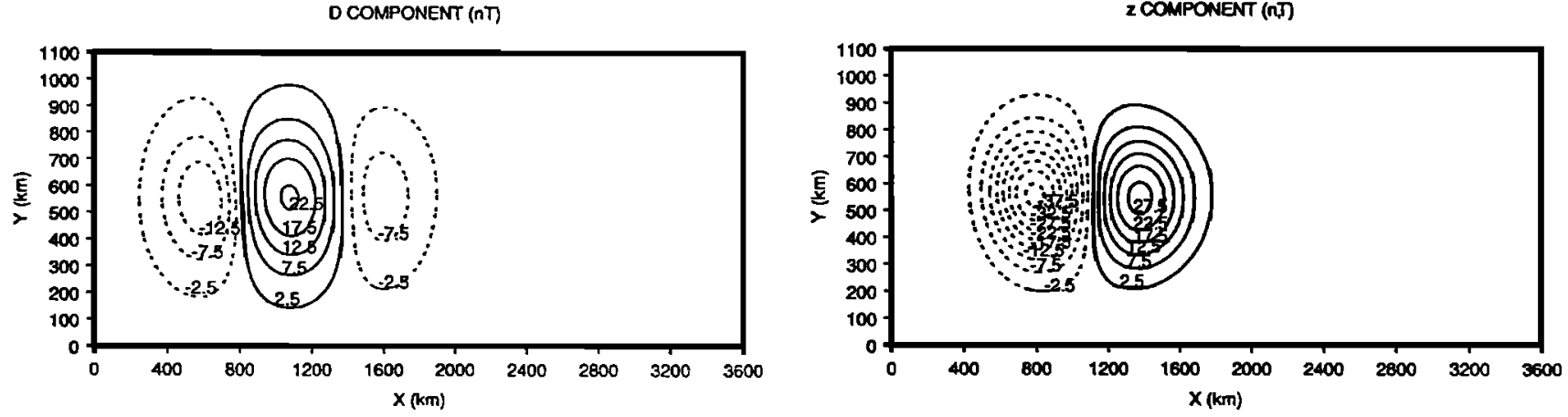

Figure 4. TCV signatures for the case in which both the conductivity enhancement and ground induction effects are included. The background ionospheric conductance decreases linearly from 3 mhos on the dayside to 1 mho on the nightside; this behavior approximates the ionospheric conditions for winter and solar maximum. The other parameters are the same as for Figure 1.

locally enhanced conductivity associated with the TCV precipitation is present.

Figure 6 shows the case in which the westward speed of the TCV is $20 \mathrm{~km} / \mathrm{s}$ and the rest of the parameters are the same as those for the case shown in Figure 5. As we mentioned in the introduction, the TCVs with speeds of higher than $20 \mathrm{~km} / \mathrm{s}$ were observed, but they are rare. The purpose of choosing the speed of $20 \mathrm{~km} / \mathrm{s}$ in the simulation is to see the maximal effect of TCVs' speed on ground signatures. First of all, because of the high speed of the TCV, the region of enhanced conductivity is greatly elongated. The elongated conductivity region causes a greater distortion of the upward current cell. At the same time the elongation of the higher conductivity region and the higher speed allows a larger portion of downward current cell to move into a high-conductivity region, thus leading to a greater distortion of the downward current cell. All these effects cause a more severe wrapping of the two current filaments, which in turn leads to a greater rotation of the whole ground magnetic disturbance patterns. Based on the above results, a useful theoretical prediction is that when TCVs with a clockwise leading convection cell move toward the nightside, a higher speed may lead to a greater asymmetry of the ground magnetic disturbance observed from a single ground-based magnetometer, because the whole magnetic pattern is rotated. The combination of the conductance enhancement and the rotation of ground magnetic patterns due to the wrapping of two current cells could lead to asymmetry of well above $30 \%$ in the TCVs' magnetic signatures observed from a single groundbased magnetometer, depending on the relative location of the magnetometer from the TCVs and the TCVs' speed.

\subsection{Triple Traveling Convection Vortices}

Recent observations have shown that triple traveling convection vortices also frequently occur in the prenoon or postnoon sectors of high-latitude ionosphere [Sibeck, 1995; 

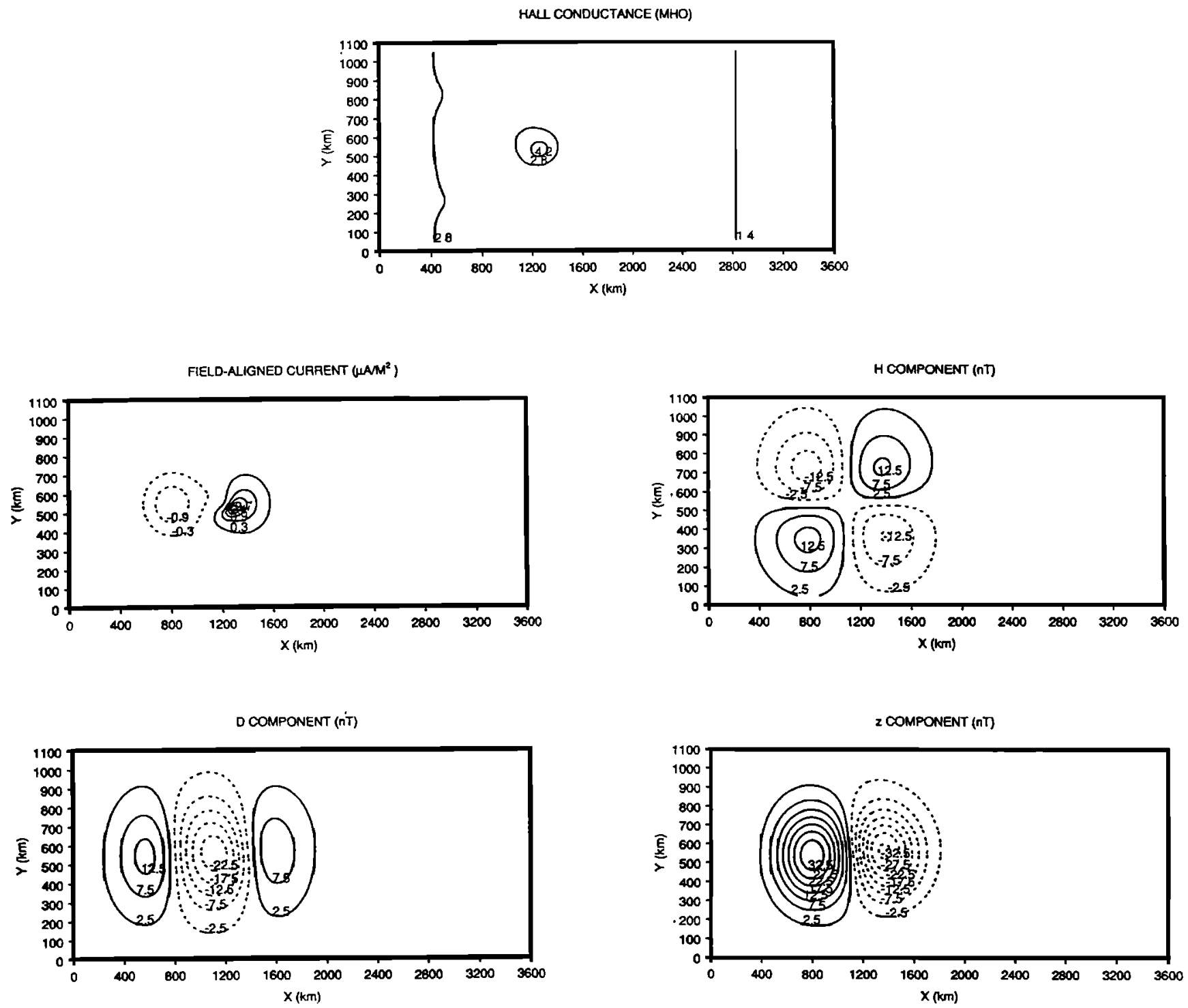

Figure 5. TCV signatures for the case in which the leading convection cell of the TCV is clockwise (upward current). The other parameters are the same as for Figure 4.

Zesta et al., 1995]. The leading convection cell can be either clockwise (upward field-aligned current) or counterclockwise (downward field-aligned current) with alternating rotation directions for neighboring cells.

Figure 7 shows the case of a triple TCV with a clockwise leading convection cell (upward current). The TCV moves toward the nightside with a constant speed of $4 \mathrm{~km} / \mathrm{s}$. We can see that in this case all three field-aligned current filaments are distorted. The difference between the ground magnetic disturbance patterns of triple and twin TCV is mainly the number of magnetic disturbance cells, with the triple TCV having an additional cell for all components. An inspection of Figure 7 indicates that there is a rotation of the magnetic disturbance patterns associated with the first two TCV cells on the west side. Obviously, this is due to the wrapping of these two field-aligned current filaments.

We also ran the case of a triple TCV with a counterclockwise (downward current) leading convection cell. As we expected, now the first two current cells on the east side tend to wrap around each other instead of the two on the west side as shown in Figure 7. The overall patterns are similar to the ones shown in Figure 7 except for a sign difference.

\section{Summary}

We have conducted a modeling study of ground magnetic signatures of traveling convection vortices that includes both the ionospheric conductivity enhancement associated with the TCVs and the ground induction effect. The major results are summarized as follows:

1. The localized conductivity enhancement associated with the TCV precipitation can cause a significant distortion of the TCV current system and leads to a distortion of the ground magnetic disturbance patterns. The patterns of all three magnetic components are asymmetric, mainly in the E-W direction, with stronger magnetic disturbances on the side of clockwise convection cells (upward field-aligned currents). The 

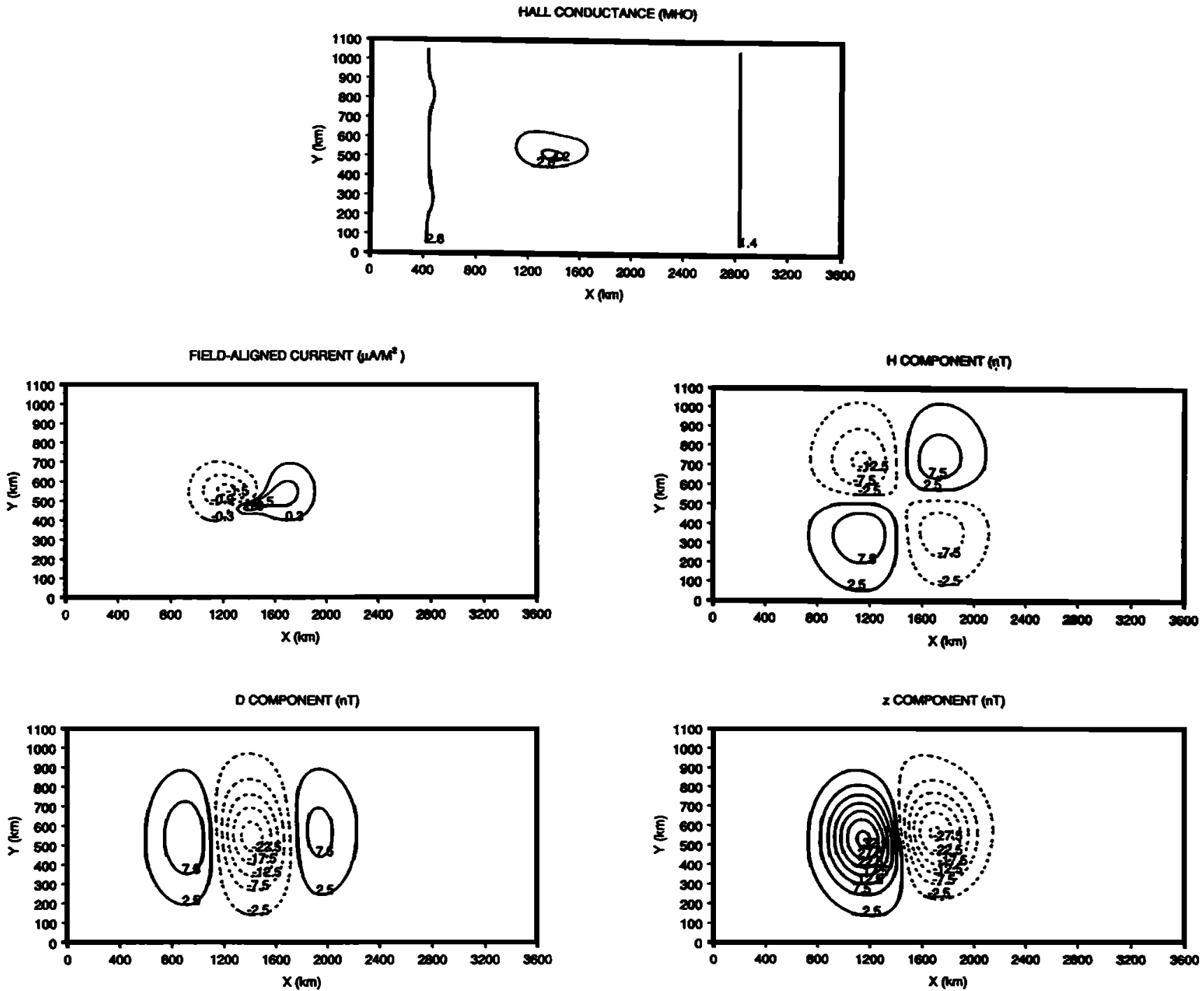

Figure 6. TCV signatures for the case in which the TCV has a high westward speed $(20 \mathrm{~km} / \mathrm{s})$. The other parameters are the same as for Figure 5.

pattern of the $Z$ component shows the strongest asymmetry (20-30\%)

2. For the case of uniform Earth conductivity the effect of induction currents on the ground magnetic signatures of the TCVs is insignificant (less than $5 \%$ ). These currents mainly weaken the disturbances in the $H$ and $D$ components and strengthen the disturbances in the $Z$ component. The effect of Earth's inhomogeneity is not explored in this work.

3. Since the E-W asymmetry in the magnetic disturbance patterns is caused by the localized conductivity enhancement associated with the TCV precipitation, we predict that a severely distorted magnetic pattern observed on the ground may indicate a TCV with hard precipitation.

4. Because of the presence of the localized conductivity enhancement the polarity and speed of the TCVs can significantly influence the distortion features of ground magnetic patterns. When a clockwise convection cell leads, the upward and downward current filaments tend to wrap around each other because of the intrusion of the counterclockwise cell into enhanced conductivity regions, which results in a rotation of the whole ground magnetic disturbance patterns. This rotation of the patterns may make a further contribution to the asymmetries of the magnetic disturbances observed by groundbased magnetometers. A higher convection speed leads to a more elongated enhanced conductivity region, which can cause a more severe wrapping of current filaments. Then, magnetic patterns with a greater rotation can be expected on the ground. The combination of the conductance enhancement and the rotation of ground magnetic patterns due to the wrapping of two current cells could lead to asymmetry of well above $30 \%$ in the TCVs' magnetic signatures observed from a single groundbased magnetometer, depending on the relative location of the magnetometer from the TCVs and the TCVs' speed.

5. The difference between the ground magnetic disturbance patterns of triple and twin TCVs is mainly the number of magnetic disturbance cells, with the triple TCVs having an additional cell for all components.

The goal of this study was not to decisively connect the observed asymmetry of ground magnetic disturbances to the conductivity enhancement and induction current effects. 


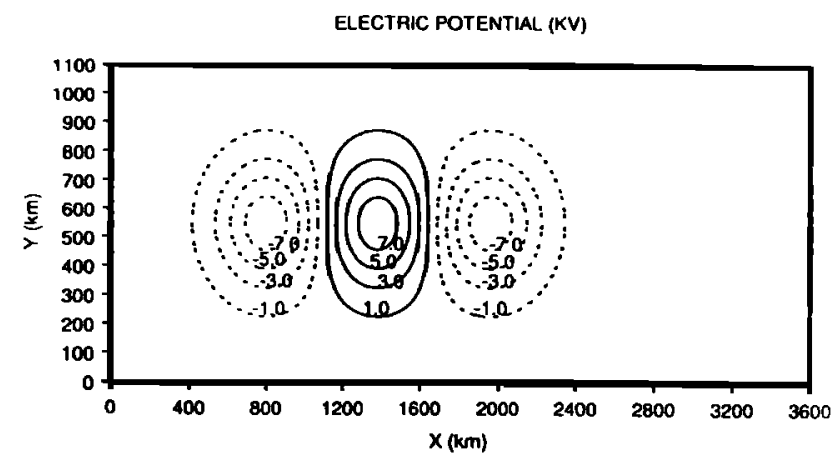

FIELD-ALIGNED CURRENT ( $\left(\mathrm{AM}^{2}\right)$

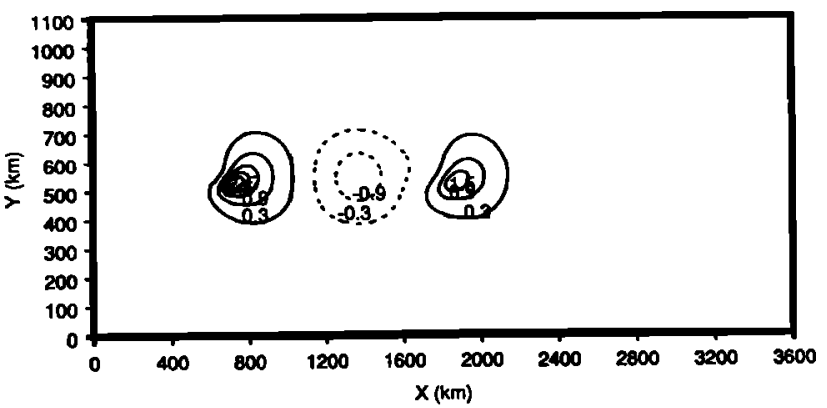

D COMPONENT (nT)

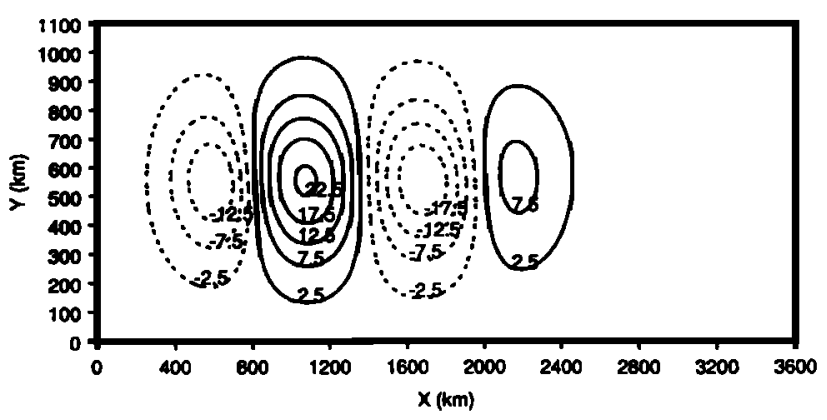

H COMPONENT (nT)

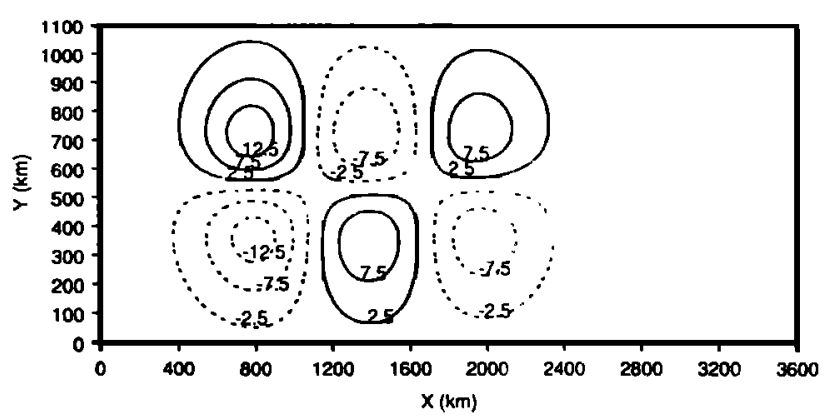

z COMPONENT (nT)

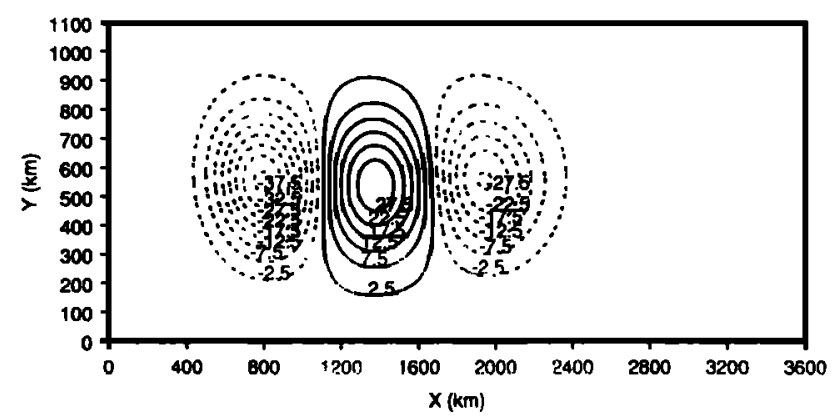

Figure 7. Signatures for a triple TCV. The leading convection cell is clockwise (upward current), and the convection speed is $4 \mathrm{~km} / \mathrm{s}$.

Instead it was to theoretically show how the localized conductivity gradient and ground induction contribute to the distortion of the ground magnetic disturbance patterns of the TCVs and how the various TCV characteristics (such as speed and polarity) affect the features of the ground magnetic patterns when the conductivity enhancement and ground induction are present. To answer the questions of whether or not the asymmetric features shown in the modeling are consistent with the observed ones and whether or not the localized conductivity gradient and the speed and polarity of the TCVs actually determine the observed asymmetry of ground magnetic disturbances, a series of one-to-one model-observation comparison studies have to be done in which a set of realistic ionospheric parameters of the TCVs are needed.

Acknowledgments. This research was supported by NASA grant NAG5-1484, NSF grant ATM-93-08163, and Naval Research grant N00014-95-1-0652 to Utah State University. We thank the two referees for their very constructive comments and suggestions.
The editor thanks W. A. Bristow and B. J. Fraser for their assistance in evaluating this paper.

\section{References}

Bristow, W. A., et al., Observations of convection vortices in the afternoon sector using the SuperDARN HF radars, J. Geophys. Res., $100,19,743,1995$.

Campbell, W. H., Electrical Properties of the Earth's Mantle, Birkhäuser Boston, Cambridge, Mass., 1987.

Chapman, S., The solar and lunar diurnal variations of terrestrial magnetism, Philos. Trans. R. Soc. London, Ser. A, 1, 218, 1919.

Chaston, C. C., H. J. Hansen, F. W. Menk, B. J. Fraser, and Y. D. Hu, Ground signatures of convecting reconnected flux tubes, J. Geophys. Res., 98, 19,151, 1993.

Fridman, M., and J. Lemaire, Relationship between auroral electrons fluxes and field-aligned potential difference, J. Geophys. Res., 85, $664,1980$.

Friis-Christensen, E., M. A. McHenry, C. R. Clauer, and S. Vennerstrom, Ionospheric traveling convection vortices observed near the polar cleft: A triggered response to sudden changes in the solar wind, Geophys. Res. Lett., 15, 253, 1988. 
Fukushima, N., Equivalence in ground geomagnetic effect of ChapmanVestine's and Birkland-Alfven's electric current-systems for polar magnetic storms, Rep. Ionos. Space Res. Jpn., 23, 219, 1969.

Glassmeier, K., Traveling magnetospheric convection twin-vortices: Observations and theory, Ann. Geophys., 10, 547, 1992.

Glassmeier, K., and C. Heppner, Traveling magnetospheric convection twin vortices: Another case study, global characteristics, and a model, J. Geophys. Res., 97, 3977, 1992.

Glassmeier, K., M. Honisch, and J. Untiedt, Ground-based and satellite observations of traveling magnetospheric convection twin vortices, $J$. Geophys. Res., 94, 2520, 1989.

Heikkila, W. J., T. S. Jorgensen, L. J. Lanzerotti, and C. G. Maclennan, A transient auroral event on the dayside, J. Geophys. Res., 94, $15,291,1989$.

Kisabeth, J. L., and G. Rostoker, Modeling of three dimensional current system associated with magnetospheric substorms, Geophys. J. R. Astron. Soc., 49, 655, 1977.

Kivelson, M. G., and D. J. Southwood, Ionospheric traveling vortex generation by solar wind buffeting of the magnetosphere, J. Geophys. Res., 96, 1661, 1991.

Lanzerotti, L. J., L. C. Lee, C. G. Maclennan, A. Wolfe, and L. V. Medford, Possible evidence of flux transfer events in the polar ionosphere, Geophys. Res. Lett., 13, 1089, 1986.

Lanzerotti, L. J., R. M. Konik, A. Wolfe, D. Venkatesan, and C. G. Maclennan, Cusp latitude magnetic impulse events, 1, Occurrence, statistics, J. Geophys. Res., 96, 14,009, 1991.

Lohr, H., and W. Blawert, Ground signatures of traveling convection vortices, in Solar Wind Sources of Magnetospheric Ultra-Low Frequency Waves. Geophys. Monogr. Ser., vol. 81, edited by M. J. Engebretson, K. Takahashi, and M. Scholer, p. 231, AGU, Washington, D.C., 1994.

Lysak, R. L., and D.-H. Lee, The response of the dipole magnetosphere to pressure pulses, Geophys. Res. Lett., 19, 937, 1992.

McHenry, M. A., and C. R. Clauer, Modeled ground magnetic signatures of flux transfer events, J. Geophys. Res., 92, 11,231, 1987.
McHenry, M. A., C. R. Clauer, E. Friis-Christensen, P. T. Newell, and J. D. Kelly, Ground observations of magnetospheric boundary layer phenomena, J. Geophys. Res., 95, 14,995, 1990a.

McHenry, M. A., C. R. Clauer, and E. Friis-Christensen, Relationship of solar wind parameters to continuous dayside, high-latitude traveling ionospheric convection vortices, J. Geophys. Res., 95, 15,007, $1990 \mathrm{~b}$.

Schunk, R. W., L. Zhu, and J. J. Sojka, Ionospheric response to traveling convection twin vortices, Geophys. Res. Lett, 21, 1759, 1994.

Sibeck, D. G., A model for the transient magnetospheric response to sudden solar wind dynamic pressure variations, J. Geophys. Res., 95, 3755, 1990.

Sibeck, D. G., Current systems associated with magnetic impulse events and traveling convection vortices (abstract), EOS Trans. AGU, 76 (46), Fall Meet. Suppl., F489, 1995.

Southwood, D. J., and M. G. Kivelson, Vortex motion in the ionosphere and nonlinear transport, J. Geophys. Res., 98, 11,459, 1993.

Vo, H. B., J. S. Murphree, R. D. Elphinstone, R. C. Elphic, E. FriisChristensen, H. Luhr, and D. G. Sibeck, Multipoint observations of a dayside transient event, J. Geophys. Res., 99, 13,409, 1994.

Wej, C. Q., and L. C. Lee, Ground magnetic signatures of moving elongated plasma clouds, J. Geophys. Res., 95, 2405, 1990.

Zesta, E., W. J. Hughes, and M. J. Engebretson, The evolution of traveling convection vortices (TCV's) (abstract), EOS Trans. AGU, 76(46), Fall Meet. Suppl., F516, 1995.

Zhu, L., and J. R. Kan, Ground magnetic signatures of multiple fieldaligned current sheets in flux transfer events, J. Geophys. Res., 94, $6655,1989$.

P. Gifford, R. W. Schunk, J. J. Sojka, and L. Zhu, Center for Atmospheric and Space Sciences, Utah State University, Logan, UT 84322-4405. (e-mail: schunk@cc.usu.edu; fasojka@sojka.cass.usu.edu; zhu@theory.cass.usu.edu)

(Received May 28, 1996; revised November 25, 1996; accepted November 25, 1996.) 